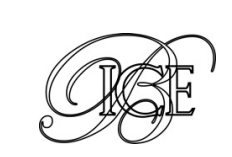

Faith Christian Q. Cacnio*

Jade Eric T. Redoblado*

Hazel C. Parcon-Santos*

Marites B. Oliva*

\title{
LA ESTRATEGIA DE FIJACIÓN DE OBJETIVOS DE INFLACIÓN EN FILIPINAS \\ Una evolución acorde con los acontecimientos mundiales
}

La estrategia de fijación de objetivos de inflación se está convirtiendo en la estrategia preferente de política monetaria. A lo largo de este artículo los autores describen la evolución de la estrategia de fijación de objetivos de inflación en Filipinas y aportan una evaluación de su impacto a la vista de la cambiante dinámica de la inflación de este país. El análisis cuantitativo muestra que la economía filipina se ha beneficiado de la adopción de una estrategia de fijación de objetivos de inflación, como prueba la caída gradual de la persistencia de la inflación y el hecho de que el proceso inflacionista esté más centrado en el futuro. Asimismo, la inflación se ha vuelto menos sensible a perturbaciones en la brecha del producto y en la del tipo de cambio real, gracias a una implementación cré́ble y eficiente de la estrategia, y se han reforzado los efectos a largo plazo de la política monetaria sobre los tipos de interés de mercado. Los resultados también indican que, debido a los crecientes desafíos internos y externos, se requiere una estrategia de fijación de objetivos de inflación un poco más flexible si se quiere lograr una estabilidad de precios compatible con un crecimiento sostenible.

Palabras clave: Filipinas, política monetaria, objetivos de inflación, tipos de interés, tipo de cambio, eficacia de la política monetaria.

Clasificación JEL: E43, E52, E58.

\section{Introducción}

Durante las dos últimas décadas se ha generado un amplio consenso sobre el hecho de que la estabilidad de precios - una tasa de inflación baja y estable- proporciona importantes beneficios a la economía. Aunque la evidencia empírica sobre el impacto de la inflación en el crecimiento económico

* Miembros del Centro de Política Monetaria y Financiera (Center for Monetary and Financial Policy) del Banco Central de Filipinas.

Las opiniones expresadas en este documento son únicamente las de los autores y no reflejan las del Banco Central de Filipinas.

Versión de marzo de 2016. varía según los países, la observación general es que la inflación, particularmente en niveles altos, puede ser perjudicial para el crecimiento. Por el contrario, una inflación baja y estable reduce las incertidumbres y distorsiones en la economía, permitiendo con ello que los agentes económicos (es decir, los hogares y empresas) gestionen mejor sus decisiones de consumo e inversión. Así, la teoría y la evidencia sugieren que la política monetaria debería centrarse en promover la estabilidad de precios (Mishkin, 2007). El fuerte énfasis en la estabilidad de precios ha dado lugar a que la estrategia de $D$ 
fijación de objetivos de inflación (inflation targeting o IT por sus siglas en inglés ${ }^{1}$ ) se haya convertido en el esquema preferido de política monetaria.

En este artículo se presenta la evolución de la estrategia de fijación de objetivos de inflación en Filipinas y se ofrece un análisis de su impacto a la vista de la evolución de la dinámica de la inflación en Filipinas. El apartado 2 discute el marco de la estrategia de fijación de objetivos de inflación y evalúa su rendimiento. En el apartado 3 se presentan los resultados de las evaluaciones cuantitativas del impacto de la estrategia de fijación de objetivos de inflación. El apartado 4 describe diversas cuestiones relacionadas con la aplicación de la estrategia de fijación de objetivos de inflación para el futuro, y el apartado 5 presenta las conclusiones.

\section{La estrategia de fijación de objetivos de inflación en Filipinas}

\subsection{El cambio hacia la fijación de objetivos de inflación}

Antes de adoptar una estrategia de fijación de objetivos de inflación, el Banco Central de Filipinas (en adelante BSP, acrónimo de su nombre oficial en tagalo de Bangko Sentral ng Pilipinas) utilizaba un enfoque de política monetaria basado en agregados monetarios. En el supuesto de que la velocidad del dinero se mantenga estable en el tiempo, los cambios en la oferta de dinero están directamente relacionados con los cambios de precios o la inflación. El enfoque fue posteriormente modificado para poner un mayor énfasis en la estabilidad de precios en vez de respetar estrictamente los objetivos fijados para los agregados monetarios, de modo que el BSP podía rebasar los objetivos monetarios siempre y cuando la tasa de inflación

\footnotetext{
1 No existe consenso en la literatura económica en español sobre la traducción de la estrategia de inflation targeting, que se recoge a veces como «fijación de objetivos de inflación» o «metas de inflación» o «inflación objetivo". Hemos optado por la primera. Tampoco se suele utilizar en español un acrónimo como el de IT, aunque en algún pasaje del artículo recurriremos a él para evitar una excesiva repetición de la expresión ( $\mathrm{N}$. del T.).
}

efectiva se mantuviera dentro de los niveles programados.

En los años ochenta y noventa se llevaron a cabo reformas para promover la liberalización del entorno del sistema financiero en Filipinas, y para hacerlo competitivo con otros mercados financieros internacionales. Sin embargo, se observó que dicha liberalización condujo al debilitamiento de la relación entre los objetivos monetarios cuantitativos y la inflación, debido a algunos «cambios estructurales» en la velocidad de circulación del dinero, y a volatilidades e inestabilidades en el multiplicador del dinero (Lim, 2008). Estas inestabilidades se atribuyeron a los cambios observados en la demanda de dinero. Con un desplazamiento de ésta, resultaba cada vez más difícil para el banco central alcanzar sus objetivos de base monetaria.

A partir de 2002 se adoptó y comenzó a aplicarse un enfoque de fijación de objetivos de inflación. En este enfoque, un banco central anuncia un objetivo de inflación y se compromete a cumplirlo en un período de tiempo especificado?

Los análisis iniciales en Filipinas indican que la adopción de una estrategia de fijación de objetivos de inflación habría contribuido a mantener un comportamiento favorable de la inflación en el medio plazo (Guinigundo, 2005). Houben (1997) señalaba que la adhesión estricta a un ancla monetaria y de tipo de cambio podría tener altos costes en términos de crecimiento de la producción o de la inflación, dada la vulnerabilidad del país a grandes perturbaciones monetarias y reales. Por otra parte, una política que estableciera un objetivo directo de inflación mejoraría la eficacia de la política monetaria mediante la inclusión del objetivo de inflación dentro la estrategia de política monetaria. Por otra $\triangleright$

\footnotetext{
2 El Gobierno nacional, a través del Comité de Coordinación de Presupuesto de Desarrollo (DBCC), establece un objetivo de inflación con una anticipación de dos años en coordinación con el BSP. El DBCC es un órgano interinstitucional responsable de establecer los objetivos anuales del Gobierno para las variables macroeconómicas (por ejemplo, el crecimiento del producto nacional bruto o PNB y del producto interno bruto o PIB) que son insumos importantes en la formulación de los ingresos, los gastos y la financiación de programas del GN. Sin embargo, la responsabilidad de lograr la meta de inflación descansa primordialmente en el BSP. La coordinación entre el BSP y el GN en la fijación de los objetivos de inflación asegura que estén alineados con los objetivos económicos del país.
} 


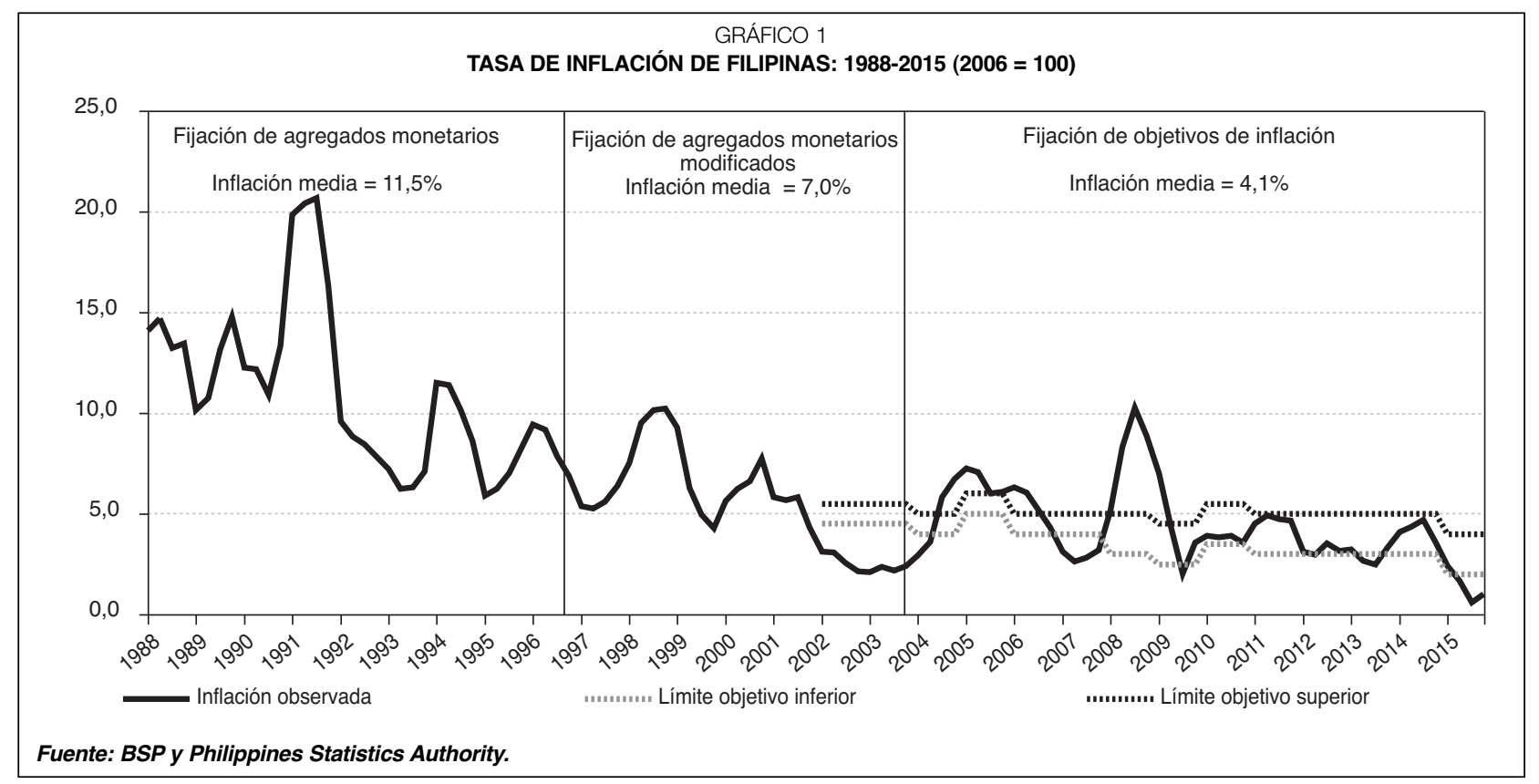

parte, Debelle y Lim (1998) observaron que el mantenimiento continuado de una estrategia basada en agregados monetarios podría hacer difícil para Filipinas alcanzar y mantener un entorno de baja inflación. Filipinas necesitaba tener credibilidad en el compromiso de una baja inflación a medio plazo, y la estrategia de fijación de objetivos de inflación se vio como una forma eficaz de lograr este objetivo. De igual manera, la estrategia de fijación de objetivos de inflación proporciona al BSP un marco de política económica coherente y consistente con un objetivo claro: la estabilidad de precios.

\subsection{Eficacia de la estrategia de fijación de objetivos de inflación}

Tras la aplicación de la estrategia de fijación de objetivos de inflación, el BSP logró controlar la inflación. Entre 1988 y 1994 la tasa media interanual de inflación general de Filipinas era del 11,5 por 100 y se redujo al 7 por 100 de 1995 a 2001, cuando el BSP adoptó una estrategia de seguimiento de agregados monetarios modificada (Gráfico 1). Después de que el BSP adoptase la estrategia de fijación de objetivos de inflación, se redujo hasta el 4,1 por 100 entre 2002 y 2015.
En los últimos años Filipinas se ha revelado como una de las economías de mejor comportamiento en el Sudeste Asiático. Se consiguió un fuerte crecimiento con una inflación dentro de niveles manejables. En 2014 el BSP logró alcanzar su objetivo de inflación por sexto año consecutivo, con una inflación promedio del 4,1 por 100 , dentro del rango objetivo de 4,0 $\pm 1,0$ por 100 . Por otra parte, la correlación entre las expectativas de inflación del sector privado y el objetivo de inflación del BSP (es decir, el punto medio) aumentó considerablemente, pasando del 0,15 en el período 2002-2008 al 0,54 en el periodo 2009-2015. Esto significa que, como el BSP logra su objetivo de inflación, las expectativas de inflación del sector privado están cada vez más ancladas al objetivo de inflación del $\mathrm{BSP}^{3}$. Para 2015, sin embargo, la inflación media se instaló en el 1,4 por 100, por debajo del rango objetivo de inflación del 3,0 $\pm 1,0$ por 100. La moderación de los precios del petróleo y la abundancia de oferta de alimentos contribuyeron en gran medida a estos bajos registros de inflación durante el año.

${ }^{3}$ Esta convergencia puede reflejar la respuesta positiva del sector privado a la estrategia de comunicación del BSP de anunciar su pronóstico de inflación, así como sus intenciones de política en el medio plazo (Guinigundo, 2016). 


\section{Evaluación del impacto de la estrategia de fijación de objetivos de inflación sobre la dinámica de la inflación ${ }^{4}$}

¿Qué factores permiten explicar el aparente éxito de la estrategia de fijación de objetivos de inflación? Levin et al. (2004) observaron que la estrategia de fijación de objetivos de inflación desempeña un papel clave en el anclaje de las expectativas de inflación y en la reducción de la persistencia de la misma. En este marco, las autoridades monetarias han de comunicar adecuadamente al público sus decisiones y acciones de política monetaria. Cuanto más informados están los agentes del mercado de los objetivos e intenciones del banco central, menos propensos se vuelven a modificar sustancialmente sus expectativas de inflación futura, incluso ante shocks de inflación transitorios (Davis, 2014). La mayor transparencia y rendición de cuentas, asociadas con el paso, en 2002, a una estrategia de fijación de objetivos de inflación incrementó la importancia del canal de las expectativas en la gestión de la política monetaria en Filipinas (Guinigundo, 2014), lo que resulta evidente a la vista de la convergencia observada entre el comportamiento de las expectativas de inflación del sector privado y la previsión de inflación del BSP entre enero de 2009 y octubre de 2014. La estrecha relación entre la política monetaria y las expectativas de los mercados permitió que el BSP ajustase sus tipos de intervención apenas cuatro veces en 2012, ninguna en 2013 , dos veces en 2014 y ninguna en $2015^{5}$.

\subsection{La curva de Phillips modificada}

Para evaluar el impacto de la estrategia de fijación de objetivos de inflación en la dinámica de la inflación en Filipinas se utilizó una curva de Phillips aumentada por expectativas.

\footnotetext{
${ }^{4}$ La discusión en esta sección sigue a la de Guinigundo (en prensa, 2016).

${ }^{5}$ El tipo de intervención a un día se redujo 100 puntos básicos en 2012 y aumentó 50 puntos básicos en 2014
}

$$
\begin{gathered}
\pi_{\dagger}=\alpha+\beta_{1} \cdot \pi_{t-1}+\left(1-\beta_{1}\right) \cdot E_{t} \pi_{t+1}+\beta_{2} \cdot \text { ygap }_{t-1}+{ }_{[1]} \\
+\beta_{3} \cdot \text { zgap }_{\dagger}+\varepsilon_{\dagger}
\end{gathered}
$$

Donde $\pi_{t}$ es la inflación trimestral, ygap una estimación de la brecha de producción (output gap) o desviación del PIB real respecto al potencial y zgap es la brecha de tipo de cambio real o (real exchange rate gap) o desviación del tipo de cambio real respecto a su tendencia. El retardo de la inflación capturará la persistencia observada en la inflación, mientras que la inflación futura capturará la influencia de las expectativas de inflación ${ }^{6}$. La variable zgap recogerá potenciales perturbaciones externas de oferta.

El análisis se centró en los cambios que se produjeron en la dinámica de la inflación de Filipinas entre los períodos 1988-2001 (período pre-IT o período previo a la fijación de objetivos de inflación) y 2002-2015 (período IT o período de estrategia de fijación de objetivos de inflación) ${ }^{7}$. De acuerdo con los resultados estimados, el coeficiente de la inflación retardada se redujo del 0,53 en el período pre-IT al 0,48 en el período de IT, mientras que el coeficiente de inflación futura aumentó de 0,47 en el período pre-IT a 0,52 en el período IT (Tabla 1). Además, el coeficiente de la brecha de producto se redujo del 0,15 en el período pre-IT al 0,12 en el período de IT, mientras que el coeficiente de la brecha de tipo de cambio se redujo de $-0,06$ en el período pre-IT a 0,01 en el período de IT. Estos resultados implican que, entre los dos períodos: a) la persistencia de la inflación ha disminuido gradualmente a medida que el proceso de inflación pasó de estar más basado en el pasado, a más basado en el futuro; b) la curva de Phillips para Filipinas parece estar aplanándose a medida que la inflación a corto plazo se ha vuelto menos sensible a $\triangleright$

\footnotetext{
6 Esto implica que la inflación actual depende en parte de una media ponderada de su futuro y los valores retardados. La suma de los coeficientes de retardo de la inflación y la inflación futura se limita a menudo a 1 (con la constante restringido a ser 0 ). Esto es para asegurar que, en el largo plazo, la curva de Phillips es vertical (es decir, el modelo "aceleracionista" de la inflación).

7 Todos los datos utilizados son series temporales trimestrales del período 1TR 1988 - 4TR 2015. La inflación se mide como la variación porcentual trimestral interanual en el IPC. La brecha de producción (output gap) y la de tipo de cambio real (real exchange rate gap) se estimaron usando el filtro HP.
} 


\begin{tabular}{|c|c|c|c|c|c|c|c|}
\hline \multicolumn{8}{|c|}{$\begin{array}{c}\text { TABLA } 1 \\
\text { RESULTADOS DE LA REGRESIÓN DE LA ECUACIÓN [1] }\end{array}$} \\
\hline Variables & Coeficiente & $\begin{array}{c}\text { 1988-2001 } \\
\text { (Período previo a } \\
\text { estrategia de IT) }\end{array}$ & Error estándar & Estadístico t & $\begin{array}{c}\text { 2002-2015 } \\
\text { (Período de } \\
\text { estrategia de IT) }\end{array}$ & Error estándar & Estadístico $t$ \\
\hline $\begin{array}{l}\text { Persistencia de la inflación } \\
\left(\pi_{\mathrm{t}-1}\right)\end{array}$ & $\beta_{1}$ & 0,53 & 0,043 & 12,455 * & 0,48 & 0,065 & 7,431 * \\
\hline $\begin{array}{l}\text { Inflación esperada } \\
\left(\pi_{t+1}\right)\end{array}$ & $\left(1-\beta_{1}\right)$ & 0,47 & 0,043 & 10,941 * & 0,52 & 0,065 & $7,957^{*}$ \\
\hline Output gap (ygap) & $\beta_{2}$ & 0,15 & 0,081 & $1,845^{* *}$ & 0,12 & 0,07 & 1,736 ** \\
\hline $\begin{array}{l}\text { Gap del tipo de cambio real } \\
\text { (zgap) }\end{array}$ & $\beta_{3}$ & $-0,06$ & 0,02 & $-3,034$ * & $-0,01$ & 0,018 & $-0,424^{*}$ \\
\hline \multicolumn{8}{|c|}{ 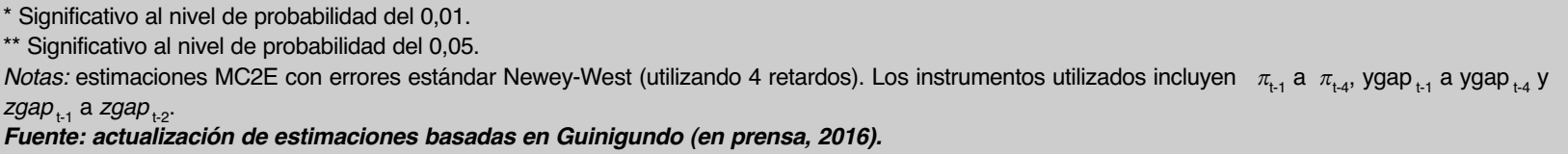 } \\
\hline
\end{tabular}

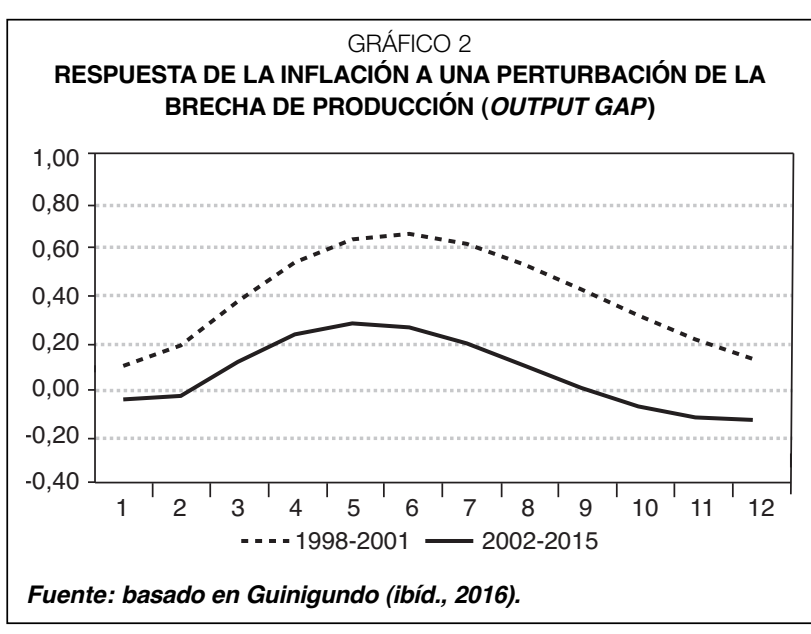

las perturbaciones de la producción o del empleo; y c) la respuesta de la inflación a corto plazo a las perturbaciones del tipo de cambio real parecen haberse reducido. Otros estudios del BSP también muestran que la significación estadística de la persistencia de la inflación ha disminuido y que ha aumentado la significación estadística de las expectativas de inflación.

Estas estimaciones son consistentes con las funciones resultantes de impulso de respuesta (impulse response functions o IRF) (Gráficos 2 y 3 ) obtenidas a partir de los modelos de vectores autorregresivos (VAR) que construimos para mostrar los efectos de una curva de oferta agregada más plana para Filipinas. Los gráficos indican que la respuesta de la inflación a perturbaciones del output gap y a perturbaciones de la desviación del tipo de cambio
GRÁFICO 3

RESPUESTA DE LA INFLACIÓN A UNA PERTURBACIÓN DE LA BRECHA DE TIPO DE CAMBIO REAL (REAL EXCHANGE RATE GAP)

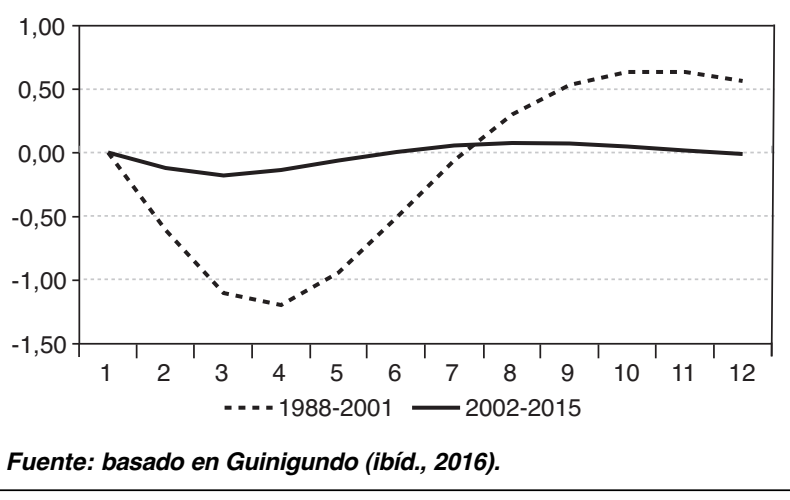

real es significativamente menor en el período de IT que en el período pre-IT. La respuesta de la inflación a perturbaciones del output gap durante el período de IT es sólo un 20 por 100 de la existente en el período pre-IT, mientras que la respuesta de la inflación a perturbaciones de la desviación del tipo de cambio real durante el período de IT es sólo un 13 por 100 de la del período pre-IT, en promedio.

En el caso de Filipinas, un curva de Phillips «más aplanada» tiene importantes implicaciones para la conducción de la política monetaria: significa no sólo que los shocks de demanda y los errores de política monetaria no se traducirán en grandes movimientos de la inflación, sino que también se requerirá un mayor sacrificio en términos de output que se necesita para bajar una inflación que está $D$ 
por encima del objetivo. Esto significa una mayor importancia en la credibilidad del BSP debido al mayor peso del compromiso del banco central con su responsabilidad primordial de la estabilidad de precios.

\subsection{Transmisión (pass-through) de los tipos de interés}

La aplicación de una estrategia de fijación de objetivos de inflación se ha traducido igualmente en un papel más importante del tipo de interés como mecanismo de transmisión de la política monetaria. Sobre la base de las estimaciones de Guinigundo (ibíd., 2016) utilizando el tipo de adquisiciones temporales a un día (Reverse Repo Rate o $\mathrm{RRP)}$ del BSP, el análisis de la transmisión a los tipos de mercado (pass-through) del tipo de interés de intervención en Filipinas (Tabla 2) indica una transmisión más acusada durante el período de fijación de objetivos de inflación que considerando un período de tiempo más largo (1986-2015). La velocidad de ajuste hacia el impacto a largo plazo también parece ser más rápida durante el período de fijación de objetivos de inflación, lo que posiblemente indica que la aplicación de las políticas que se centran en el logro de objetivos de inflación puede haber ayudado a aumentar la velocidad de transmisión a los tipos de interés de mercado. Guinigundo (ibíd., 2016) también observó que, entre los diferentes tipos de mercado, la transmisión a largo plazo es mayor para los tipos a más largo plazo (por ejemplo, Bonos del Tesoro a 5 años y 10 años), seguido de los tipos de los Bonos del Tesoro a 91 días y 364 días, los de los préstamos bancarios, y los tipos de los depósitos a plazo y de ahorro. Sin embargo, Guinigundo (ibíd., 2016) observó que la trasmisión media inmediata durante el período de fijación de objetivos de inflación es menos completa que la trasmisión media a largo plazo. Por otra parte, durante el período de fijación de objetivos de inflación se estima una transmisión inmediata negativa al tipo los de préstamos bancarios, lo cual indica una aparente desconexión entre el tipo de adquisiciones temporales a un día (Reverse Repo Rate o RRP) del BSP, y el de los préstamos bancarios a la hora de fijar los precios de dichos préstamos ${ }^{8}$.

Por otra parte, hay que tener en cuenta la posibilidad de que los bancos hayan cambiado su tipo de interés de referencia pasando del tipo de intervención a un día, al tipo de las Cuentas Especiales de Depósito (Special Deposit Accounts o SDA) ${ }^{9}$, debido posiblemente al hecho de que la tenencia de efectivo y depósitos en el BSP por parte de los bancos ha crecido significativamente desde $2008^{10}$. Para ello Guinigundo (2015) utiliza un tipo de intervención ponderado (media ponderada del tipo de intervención a un día y del tipo de las SDA) para calcular la trasmisión de los tipos de interés de intervención a los tipos de interés de mercado entre 1991 y 2015. Los resultados de Guinigundo (ibíd., 2016) siguen mostrando una transmisión mayor y más rápida en promedio entre 1991 y 2015, aunque se observa una mayor transmisión media a largo plazo durante el período de fijación de objetivos de inflación. Distinguiendo entre tipos de interés de mercado, los tipos de los depósitos de ahorro y de préstamos bancarios parecen tener transmisiones incompletas a largo plazo y un ajuste más lento, incluso durante el período de fijación de objetivos de inflación, tras la reducción del tipo de intervención en $2012^{11}$.

Partiendo de la base de los resultados de Guinigundo (ibíd., 2016), hay tres posibles razones para la debilidad observada en la transmisión inmediata: a) las menores expectativas de inflación; b) la fuerte correlación percibida entre los tipos de los $\triangleright$

\footnotetext{
${ }^{8}$ Los resultados también muestran que hay una transmisión inmediata negativa a los tipos de los Bonos del Tesoro a 5 y 10 años. Sin embargo, el $R^{2}$ ajustado no es significativo.

9 Las cuentas especiales de depósito (SDA) consisten en depósitos a plazo fijo de bancos y entidades de confianza de los bancos e instituciones financieras no bancarias con el BSP. Fue introducido en noviembre de 1998 para que el BSP pudiera expandir su conjunto de herramientas de gestión de la liquidez.

10 Esto implica que los bancos, a resultas de la incertidumbre tras la gran crisis financiera mundial de 2008, han decidido invertir en activos de mayor rendimiento y más seguros. Estos incluyen los depósitos de los bancos con el BSP bajo la línea de la SDA. A partir de finales de marzo de 2016, el saldo pendiente de SDA ascendía a un billón de pesos filipinos.

11 La tasa de intervención a un día se redujo 100 puntos básicos en 2012 y aumentó 50 puntos básicos en el año 2014 .
} 


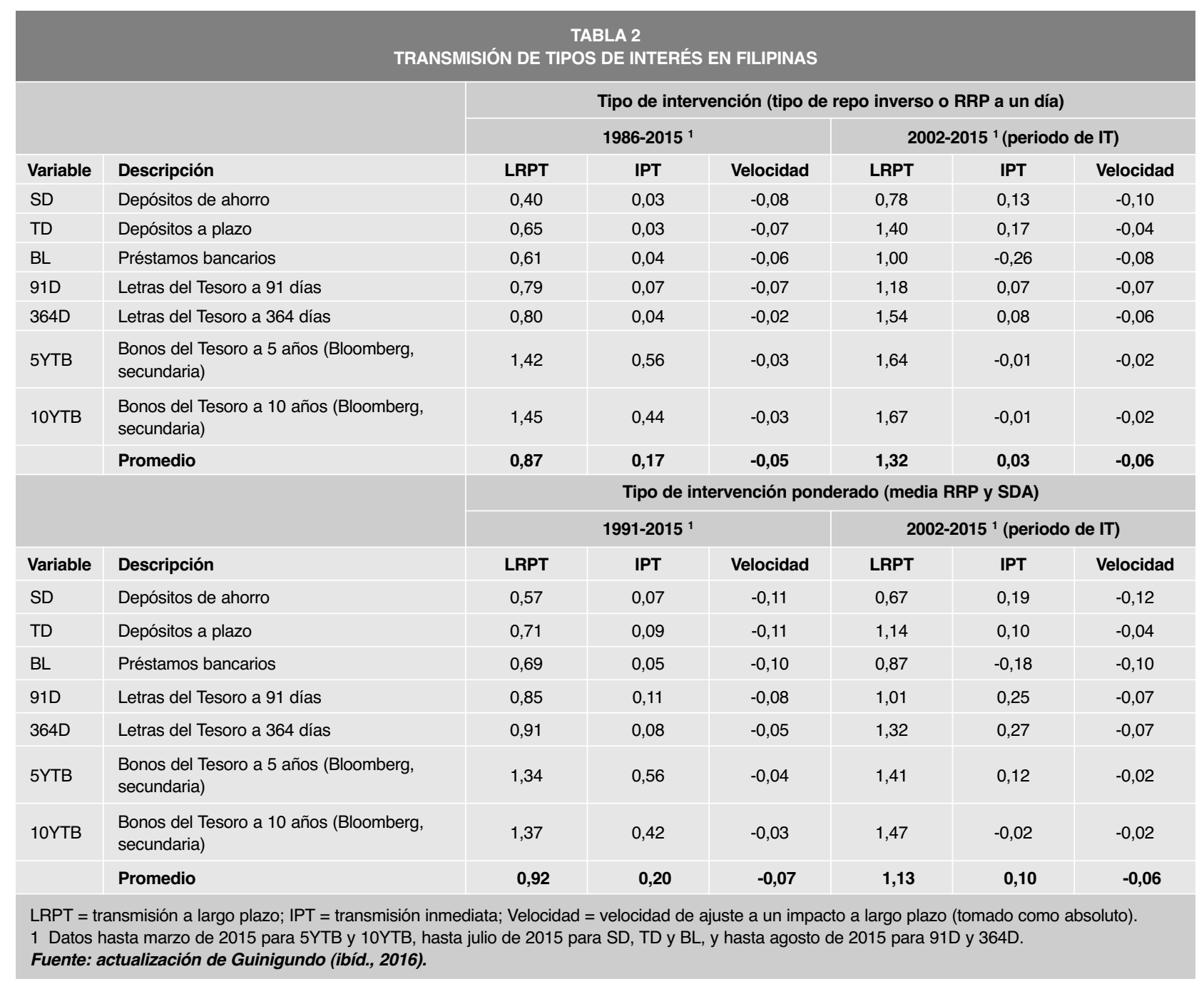

bonos a largo plazo en todos los países después de aumentos repentinos de los flujos de capital ${ }^{12}$, en el caso de tipos de interés de bonos a largo plazo; y c) un mayor efecto de las volatilidades en el mercado en vencimientos más cortos. En cuanto al primer motivo, con expectativas de inflación estables, hay momentos en los que las respuestas a las variaciones del tipo de intervención se consideran parciales, de modo que los aumentos y disminuciones en los tipos de intervención serían percibidos como algo temporal, y con alta probabilidad de retornar a la media o a los niveles normales. En

\footnotetext{
${ }^{12}$ El volumen de flujos de capital en porcentaje del PIB ha pasado del 2,5 por 100 (2005 a 2008) a 3,1 por 100 (2009 a 2014). La inversión extranjera directa y de cartera promedio atrajo alrededor de 1,4 por 100 y 1,3 por 100 del PIB nominal de 2005 a 2014, respectivamente. La mayoría de las inversiones extranjeras directas y las inversiones de cartera son instrumentos de deuda, mientras que otras inversiones se componen principalmente de préstamos.
}

el segundo caso, diversos estudios sugieren que los factores monetarios globales han afectado a los rendimientos de los bonos nacionales a largo plazo después de la gran crisis financiera. Además, los rendimientos nacionales se han vinculado más estrechamente a los rendimientos en los principales centros financieros debido a la profundización de los mercados de bonos nacionales y al aumento de la participación extranjera. Esta evolución podría dar lugar a una política monetaria menos eficaz, ya que una elevación de los tipos de intervención no aumentaría necesariamente los tipos de interés a más largo plazo. Entretanto, como el BSP apenas ajustó sus tipos de intervención en cuatro ocasiones en 2012 y un par de veces en el año 2014, los cambios en otros factores que afectan el extremo más corto de la curva de tipos podrían reducir el impacto de $\triangle$ 
las alteraciones de los tipos de intervención. Sin embargo, Guinigundo (ibíd., 2016) ha señalado que el impacto de los cambios en los tipos de intervención tendrían un impacto acumulativo en los plazos más largos, debido a los retrasos en la transmisión de la política, lo que elevaría la transmisión registrada a plazos más largos.

\subsection{Repercusión en precios (pass-through) de los tipos de cambio}

La menor respuesta estimada de la tasa de inflación a perturbaciones en la brecha del tipo de cambio real (real exchange rate gap) indica posiblemente una disminución de la repercusión en precios del tipo de cambio (ERPT o exchange rate pass-through en sus siglas en inglés) en Filipinas. En la literatura se observa, en general, una disminución de la repercusión de tipo de cambio tras la crisis financiera global. Esto pone de relieve una mayor influencia de la integración económica y financiera y sus implicaciones sobre la gestión de las políticas públicas de los bancos centrales, autoridades fiscales y reguladores del comercio. En Filipinas, una de las posibles razones de la disminución en la ERPT es la relajación de las normas cambiarias que ha ayudado a movilizar capital extranjero para financiar las necesidades de la economía doméstica (Bayangos, et al., 2016).

Para estimar la repercusión sobre la inflación del tipo de cambio se estima la ecuación siguiente:

$$
\begin{gathered}
\pi_{\dagger}^{\text {PHL }}=\mathrm{C}+\sum_{\mathrm{j}=1}^{4} \beta_{\mathrm{lj}} \pi_{t-\mathrm{j}}^{\mathrm{PHL}}+\sum_{\mathrm{k}=0}^{4} \beta_{2 \mathrm{k}} \Delta \mathrm{e}_{t-\mathrm{k}}+ \\
+\sum_{k=0}^{4} \beta_{3 k} \pi_{t-k}^{\text {world commodities }}+\sum_{k=0}^{4} \beta_{4 k} \text { ygap }_{t-k}+\varepsilon_{t}
\end{gathered}
$$

donde $\pi_{t}^{P H L}$ es la tasa de inflación interna, $\pi_{t-j}^{P H L}$ es la tasa de inflación interna retardada para controlar el impacto de la persistencia de la inflación, $\Delta e_{t-k}$ es la variación actual y retardada del tipo de cambio

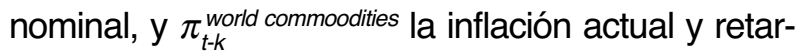
dada de las materias primas mundiales (por ejemplo, el arroz y el petróleo crudo) para aislar el impacto de cambios de precios extranjeros de los efectos de los movimientos del tipo de cambio sobre la inflación, e ygap $_{t-k}$ es la brecha de producción actual y retardada para controlar el impacto de la demanda sobre la inflación ${ }^{13}$.

El análisis se centró en la repercusión en la inflación del tipo de cambio entre dos períodos, 19882001 (o período pre-IT) y 2002-2015 (o período de $\mathrm{IT})^{14}$. Los IRF muestran una respuesta significativamente más baja de la inflación a las perturbaciones en las variaciones del tipo de cambio periodo de IT en comparación con el período pre-IT (Gráfico 4).

En este mismo sentido, Mihaljek y Klau (2008) también encontraron una disminución sustancial en la repercusión del tipo de cambio de Filipinas después de 2001. Esto podría atribuirse a la disminución del valor «señal» del tipo de cambio cuando creció la confianza en la capacidad del BSP para mantener la inflación dentro de niveles manejables. Dado que los precios internos están marginalmente afectados por las fluctuaciones del tipo de cambio, estos resultados implican que hay una mayor flexibilidad por parte de las autoridades monetarias a la hora de mantener la estabilidad de precios.

\section{Avanzando hacia una estrategia flexible de objetivos de inflación}

\subsection{Banda de tipos de interés}

Como se discutió anteriormente, los tipos de intervención tienen mayor poder en una estrategia de fijación de objetivos de inflación. No obstante, hay margen para fortalecer aún más la transmisión de la política monetaria. Como parte de sus iniciativas para establecer de manera anticipada políticas de futuro, tales como ajustes en los tipos de intervención, el BSP implementará una banda de tipos de interés (interest rate corridor o IRC) en 2016. El $\triangleright$

\footnotetext{
${ }^{13}$ La brecha de producción o output gap se estima como la producción real menos la producción potencial dividido por la producción potencial, donde se estima la producción potencial utilizando el filtro HP. La inflación se mide como la media trimestral de la variación porcentual mensual interanual del IPC.

14 Uso de datos trimestrales de series temporales para el período 1TR 1990 - 3TR 2015. La inflación se mide como la variación porcentual interanual trimestral del IPC.
} 


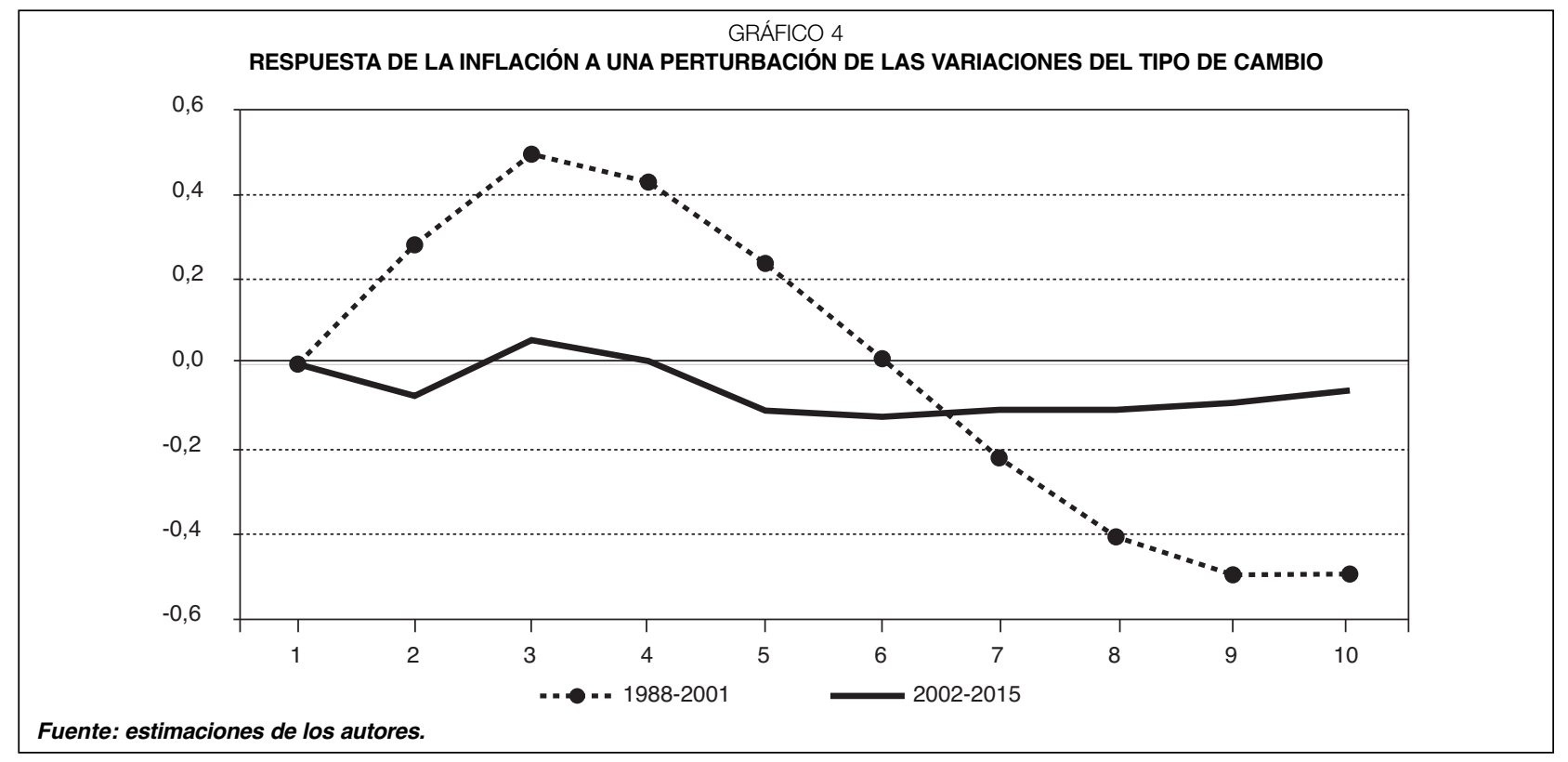

IRC es un sistema para controlar los tipos del mercado monetario hacia la tasa objetivo o de intervención del banco central. Se compone de un tipo al que el banco central presta reservas a los bancos comerciales (tipo activo) y un tipo al que aceptará sus depósitos (tipo de depósito). Por lo general, el tipo de préstamos estará por encima y el tipo de depósito por debajo del tipo objetivo o de intervención, formando una banda o corredor alrededor de éste. Con un sistema IRC se refuerza la transmisión de la política monetaria, asegurando que los tipos de interés del mercado monetario se mueven dentro de un rango razonable alrededor del tipo de interés oficial del BSP ${ }^{15}$. El IRC también ayudará al desarrollo de los mercados de capital de Filipinas mediante el fomento de las transacciones del mercado monetario y la gestión activa de la liquidez de los bancos filipinos. El aumento de la actividad en el mercado de dinero, a su vez, ayudará a promover la normalización de la curva de tipos en el país ${ }^{16,}$

15 El BSP utilizará la Facilidad de Subasta de Depósitos a Plazo (TDF), una herramienta común de los bancos centrales para la gestión de la liquidez. La TDF es adecuado para los plazos más cortos y no es apropiado para valores negociables.

${ }^{16}$ En el momento apropiado el BSP consultará a los agentes del mercado y las contrapartes para asegurar la correcta ejecución de la IRC. EI BSP también dará al mercado tiempo suficiente para prepararse para la transición.

\subsection{Estabilidad financiera}

La evolución reciente de los mercados financieros (como la crisis financiera mundial, la orientación asíncrona de la política monetaria de los bancos centrales influyentes, y el alza continua de los tipos de interés en Estados Unidos, entre otros) han dado lugar a un replanteamiento de los objetivos de los bancos centrales y de su ámbito de trabajo, en particular respecto a la estabilidad financiera. Así, una serie de bancos centrales de mercados emergentes (incluido el BSP) han implementado regulaciones macroprudenciales que han complementado las acciones de política monetaria. Tales acciones aseguran la defensa preventiva de la banca del país y del sistema financiero frente a los riesgos y las vulnerabilidades que podrían interrumpir las operaciones individuales o sistémicas de las instituciones financieras.

No obstante, sigue habiendo dudas sobre la coordinación de las medidas macroprudenciales y las políticas monetarias. Mientras que el uso de los tipos de intervención tiene repercusiones en la distribución, estas consecuencias son mucho más notables en el caso de las regulaciones macroprudenciales, como la ratio préstamo-valor (loan-tovalue ratio). Y así como los bancos centrales deben conservar su plena independencia en la conducción de la política monetaria, este no es el $\triangleright$ 
caso de herramientas macroprudenciales (Blanchard, 2015). La regulación y coordinación de la supervisión de aspectos de la banca, seguros, valores y regulaciones de cambio que deben cumplir las empresas son necesarias a fin de garantizar la coherencia y la exhaustividad de las medidas macroprudenciales. En el futuro, estas son consideraciones importantes de cara al uso de objetivos de inflación como estrategia de política monetaria.

\subsection{Mejoras en la comunicación}

El BSP está mejorando continuamente la forma en que comunica su valoración de la situación actual y de las perspectivas económicas. Como los bancos centrales reconocen la necesidad de una mayor flexibilidad, hay mayores presiones para una comunicación más eficaz en gestionar las expectativas, no sólo las de inflación, sino también de la aversión al riesgo. Esto requeriría un mayor grado de «matices» de transparencia que no amplifique las expectativas del mercado (es decir, que sea no propagandista), sino que proporcione estabilidad y seguridad en la formulación de políticas disciplinadas. De hecho, el BSP ha comenzado ya en 2015 un plan de comunicación en toda la empresa para sistematizar mejor el control de acontecimientos y la comunicación al mercado. Con ello se espera mejorar aún más la potencia y la eficacia de los instrumentos de política monetaria del BSP.

\section{Conclusiones}

Las evaluaciones cuantitativas anteriores demuestran los beneficios que la economía de Filipinas ha obtenido con la implementación de una estrategia de fijación de objetivos de inflación. En primer lugar, la persistencia de la inflación disminuyó gradualmente entre el período anterior y el posterior a la fijación de objetivos de inflación. Esto se atribuye a un cambio en el proceso de inflación, que ha pasado de ser retrospectivo a ser más prospectivo. En segundo lugar, la aplicación creíble y eficiente de los objetivos de inflación ha llevado a una menor sensibilidad (volatilidad) de la inflación a las perturbaciones en la brecha de producción y en brecha de tipo de cambio real (es decir, a un aplanamiento de la curva de Phillips). En tercer lugar, las estimaciones de las transferencias de tipos de interés indican unos efectos a largo plazo más fuertes de la política monetaria sobre los tipos de interés de mercado durante el período de estrategia de fijación de objetivos de inflación. Estos resultados son robustos a través de los modelos. Por otra parte, estos resultados indican que un enfoque más flexible para los objetivos de inflación es de vital importancia para lograr una estabilidad de precios que favorezca un crecimiento sostenible de cara a los desafíos internos y externos a la estabilidad de precios que han modificado la dinámica y la realización de la fijación de objetivos de inflación.

Esta conclusión adquiere mayor importancia en vista de la creciente integración y la evolución de la economía mundial en otros países, que han tenido efectos desbordamiento sobre la economía nacional. En particular, la reciente volatilidad en el movimiento de los flujos de capital extranjero ha sido una de las principales preocupaciones para muchos bancos centrales en los últimos años, en particular los bancos centrales de los mercados emergentes. Con el aumento de los tipos de interés en Estados Unidos, los flujos de capital han salido de los mercados emergentes y han regresado a las economías avanzadas. Estos flujos de capital han provocado, a su vez, movimientos en los tipos de cambio, así como en los precios de los activos financieros. Mientras el BSP siga un sistema de tipo de cambio determinado por el mercado, coherente con la consecución de objetivos de inflación, el BSP mantiene su presencia en el mercado de divisas durante los períodos de inusual volatilidad para mantener los movimientos alineados con los fundamentales y con los de nuestros socios regionales.

Por otra parte, el BSP ha puesto en marcha medidas macroprudenciales para evitar la acumulación de riesgos excesivos que podrían amenazar la estabilidad financiera. Asimismo, el BSP se $D$ 
coordina con otros reguladores en la promoción de unas condiciones estables para el sistema financiero y en el establecimiento de unas bases más fuertes para los dinámicos mercados financieros y de capitales. Como resultado, el sistema bancario de Filipinas se ha mantenido sólido y estable. Sin embargo, el BSP permanece vigilante ante los acontecimientos globales para que pueda reaccionar de forma preventiva a los problemas potenciales.

De cara al futuro, el BSP está comprometido con asegurar que la inflación se mantenga dentro de un rango objetivo que sea propicio para un crecimiento sostenible e integrador. Unida a las reformas fiscales y a los sólidos fundamentos macroeconómicos, el logro de la estabilidad de precios ha permitido a la economía superar las turbulencias del entorno financiero mundial.

\section{Bibliografía}

[1] BAYANGOS, V.; ELLOSO, L.; HALLIG, J.M.R.; YEUNG, J. N. y SALAMATIN, A.M. (2015). «The Impact of Foreign Exchange Liberalization Reforms on the Philippine Economy: An Initial Assessment». BSP Working Paper (en prensa).

[2] BLANCHARD, O. (2015). «Ten take aways from the 'Rethinking Macro Policy: Progress or Confusion?». Diponible en:

http://blog-imfdirect.imf.org/2015/05/01/ten-takeaways-from-the rethinking-macro-policy-progressor-confusion

[3] DAVIS, P. (2014). «Inflation targeting and the anchoring of inflation expectations: cross-country evidence from consensus forecasts». Federal Reserve Bank of Dallas Globalization and Monetary Policy Institute Working Paper, $\mathrm{n}^{\circ} 174$.

[4] DEBELLE, G. y LIM, C.H. (1998). «Preliminary Considerations of an Inflation Targeting Framework for the Philippines». IMF Working Paper 98/39.

[5] GUINIGUNDO, D. C. (2005). «Inflation Targeting: The Philippine Experience». in V. Valdepeñas Jr. (ed.), The Bangko Sentral ng Pilipinas and the Philippine Economy, Manila, Philippines: Bangko Sentral ng Pilipinas.

[6] GUINIGUNDO, D.C. (2014). «What have emerging market central banks learned about the international transmission of monetary policy in recent years? The Philippine case». BIS Papers, $\mathrm{n}^{\circ} 78$.

[7] GUINIGUNDO, D. (2015). «Increasing Financial Intermediation in the Philippines: Some Implications for Monetary Policy». Bank for International Settlements Emerging Markets Deputy Governors Meeting, 26-27 de febrero.

[8] GUINIGUNDO, D. (en prensa, 2016). «Towards a flexible inflation targeting in the Philippines». BSP Book Project.

[9] HOUBEN, A.C.F.J. (1997). «Exchange Rate Policy and Monetary Strategy Options in the Philippines: The Search for Stability and Sustainability». International Monetary Fund (IMF) Paper on Policy Analysis and Assessment 97/4.

[10] LEVIN, A.; NATALUCCI, F. y PIGER, J. (2004). "The macroeconomic effects of inflation targeting". Federal Reserve Bank of St. Louis Review, pp. 5180.

[11] LIM, J. A. (2008). «Central Banking in the Philippines: From Inflation Targeting to Financing Development». International Review of Applied Economics, vol. 22, $\mathrm{n}^{\circ}$ 2, pp. 271-285.

[12] MISHKIN, F. (2007). Monetary Policy Strategy. Cambridge: The MIT Press. 
